\title{
Quelles investigations, le non-spécialiste doit-il demander devant un patient consultant pour un dysfonctionnement érectile?
}

\author{
Pierre BONDIL
}

Service Urologie-Andrologie, Chambéry

\begin{abstract}
RESUME
La difficulté réelle d'un diagnostic précis et l'efficacité actuelle des médicaments n'ont pas remis en question, bien au contraire, l'intérêt du bilan étiologique d'un dysfonctionnement érectile (DE). Indispensable, il permet de reconnaître : a) les étiologies bénéficiant d'une prise en charge spécifique, b) le profil de risque de $D E$, c) les patients à moins bon pronostic, d) les situations à risque pour la santé et/ou des pathologies non ou mal prises en charge. L'approche du DE a été profondément modifiée par la mise en évidence que le DE est un marqueur pertinent de la qualité de vie (DE maladie) et aussi un symptôme clinique (DE symptôme) de multiples situations à risque ou de pathologies chroniques. Ainsi, l'existence ou non d'un retentissement sur la qualité de vie du patient (et du couple) orientera vers un bilan et une prise en charge de la santé sexuelle et/ou physique et/ou psychique.
\end{abstract}

Ce concept $D E$ maladie/DE symptôme explique la réduction des investigations uniquement centrées sur la verge au profit d'une évaluation globale des multiples facteurs biomédicaux et environnementaux susceptibles d'affecter l'état de "bonne santé". En pratique, la survenue d'un DE n'est jamais anodine car elle impose un premier bilan (toujours clinique et souvent biologique) accessible à tout médecin en premier contact quelle que soit sa qualification. L'objectif de ce bilan de " débrouillage " est de distinguer les cas simples (qui relèvent d'une large majorité de médecins) ou plus complexes.

En cas d'anomalies spécifiques et/ou d'échec de la première prise en charge, un bilan plus spécialisé, relevant ou non du soignant familiarisé à la prise en charge du $D E$, est indiqué. Très schématiquement: 1) avant 35 ans, le DE reflète d'abord un trouble psychologique/relationnel qui nécessite une évaluation sexo-psychologique (voire psychiatrique) d'autant plus que le DE est primaire ou ancien, 2) après 35 ans, le DE est aussi et d'abord, un signe vasculaire puis, de diverses morbidités chroniques d'où la nécessité d'un bilan somatique parallèle, 3) après
50 ans, un bilan à visée urologique et hormonal ("andropause") doit être rajouté. L'analyse systématique du profil de risque permet ainsi d'inscrire le bilan de tout homme consultant pour un DE dans une démarche éthique de service rendu au patient, mais aussi volontaire et collective de santé publique.

Mots clés : dysfonctionnement érectile, diagnostic, bilan, exploration, étiologie, dépistage cardiovasculaire

\section{INTRODUCTION}

Le dysfonctionnement érectile (DE) est défini comme "l'incapacité d'un homme à obtenir ou maintenir une érection pénienne suffisante pour permettre une relation sexuelle satisfaisante depuis au moins trois mois » $[6,19$, $34,44]$. Jusqu'en 1998, sa prise en charge ne concernait qu'un nombre réduit de spécialistes. En quelques années, le Viagra ${ }^{\circledR}$ a introduit la sexualité dans le grand public et dans la médecine, notamment chez le médecin généraliste d'où une médicalisation croissante de la sexualité et de ses troubles [13]. II a permis de libérer la parole collective sur la sexualité et ses troubles mais non individuelle. La prise en charge des troubles sexuels reste trop patient et soignant dépendante. En effet, la méconnaissance et les tabous de ce domaine de l'intimité sont encore largement partagés par les médecins et les patients.

\section{Correspondance :}

Dr Pierre BONDIL - Service d'urologie-andrologie, Centre hospitalier, Square Massalaz, Chambéry. France 73011 Tel 04.79.96.51.42 - Fax 04.79.96.51.99 Email pierre.bondil@ch-chambery.fr 
Quoique son diagnostic soit avant tout clinique, le DE est encore trop souvent un "symptôme du silence". Cela explique une prise en charge encore insuffisante car les patients (de plus en plus demandeurs) consultent souvent pour un autre problème en attendant que leur médecin leur en parle et... des médecins plus réactifs qu'actifs car mal à l'aise $[6,10,12,13,24,56]$. Ce décalage médecin patient reflète les lacunes d'informations sur la santé sexuelle qui est pourtant, avec la santé physique et psychique, un des trois éléments de la "bonne" santé selon l'OMS $[24,34]$ et un paramètre important de la qualité de vie $[10,13]$. Or, la prise en charge optimale du DE nécessite, comme pour toute pathologie, une compétence clinique, c'est-à-dire, l'intégration judicieuse des données cliniques et paracliniques avec les souhaits du malade dans la prise de décisions $[3,10,12,13,34,43,56]$.

Compte tenu des exigences actuelles d'une démarche scientifique de qualité (médecine fondée sur les preuves), du devoir d'information et de service rendu au patient, quel bilan le non spécialiste du DE peut-il et doit-il demander, une fois le diagnostic de DE établi ?

\section{Que nous apprend l'historique du bilan d'un dys- fonctionnement érectile?}

La place des investigations complémentaires a connu la même révolution $[1,4,15,18,34,35,53]$ que le traitement du DE. Le passage de la médecine fondée sur l'avis d'experts (Authority Based Medecine) à la médecine fondée sur les preuves (Evidence Based Medecine) permet de distinguer trois périodes:

1) les années $1980-94$ avec la croyance erronée d'obtenir des diagnostics étiologiques précis en multipliant les investigations (notamment sur les organes érectiles) [1] ;

2) Les années 1994-1999 avec la remise en question de l'utilité du bilan étiologique du fait d'une triple conjonction: a) la découverte de traitements pharmacologiques (AMM en 1994 pour la prostaglandine E1 en injection intracaverneuse puis en 1998 pour le sildénafil) non spécifiques mais très efficaces (jusqu'à près de $80 \%$ des sujets "tout venant" consultant pour $\mathrm{DE})[48,55]$; b) la difficulté du diagnostic étiologique ; c) le petit nombre d'étiologies bénéficiant d'un traitement spécifique. Ainsi, une enquête américaine de 2002 montrait que $80 \%$ d'urologues s'occupant de DE, utilisaient le sildénafil comme "pharmacotest oral" diagnostique et thérapeutique de première intention [43].

3) les années 2000 avec une modification profonde des objectifs du bilan $[6,10,35,56]$. On a enfin compris que la verge ne devait pas être "l'arbre qui cache la forêt" mais le témoin de l'action du cerveau, premier organe sexuel de l'homme et de la femme car véritable "gestionnaire" de la vie intime sexuelle. Cette relation verge-cerveau n'est pas à sens unique mais toujours interactive sous l'influence complexe de paramètres biomédicaux (propres au sujet) et aussi (et parfois surtout) psychologiques et/ou socio-environnementaux (souvent indépendants du sujet) car "aucun homme n'est une île". Ces progrès ont légitimé l'accord professionnel actuel $[6,12,18,22,34,35,44,56]$ de : a) limiter la prescription d'examens paracliniques spécifiques vu le manque d'intérêt ou de fiabilité d'un bon nombre, b) donner la prépondérance à la clinique pour une approche diagnostique et thérapeutique globale du patient et de son environnement.

\section{Quel est le rationnel actuel du bilan d'un dysfonc- tionnement érectile?}

a) L'approche du $D E$ a été modifiée par trois nouveaux concepts $[5,6]$ : le concept du DE "maladie" se fonde (Figure 1) sur un mal-être (reflet de l'impact négatif sur la qualité de vie de l'homme et de sa partenaire) souvent masqué et commun au couple $[8,10,11,46]$ car dans la vie quotidienne, la sexualité est plus domestique qu'exotique (au sens littéral). Néanmoins, nombre d'hommes et de femmes s'en accommodent ou se résignent d'emblée ou plus souvent, avec le temps et l'âge car la fragilité et la vulnérabilité font partie de la nature humaine et savoir les assumer appartient à la dignité d'être Homme. En pratique, toute plainte, toute demande, tout constat d'une souffrance liés au trouble sexuel impliquent un bilan spécifique de la santé sexuelle car l'amélioration de la vie sexuelle s'accompagne souvent d'un mieux-être global de l'homme et du couple $[7,8,10,11,13,56]$. La présence ou non de facteurs de gravité propres au sujet (anérection totale, $D E$ ancienne, troubles psychologiques manifestes, dépression, troubles sexuels multiples, primaires ou chez le sujet jeune) ou dépendants de son environnement (contexte de veuvage, de divorce, de conflits conjugal, familial, professionnel) a surtout un intérêt en cas de "DE maladie". Par contre, leur identification modifie la prise en charge en distinguant les cas simples, qui relèvent d'une large majorité de soignants, ou compliqués, qui nécessitent alors une approche spécifique et la demande, d'emblée ou rapidement, des avis complémentaires (relevant ou non du spécialiste du DE) d'autant plus que le DE peut n'être qu'un symptôme d'un autre trouble sexuel (éjaculation prématurée, troubles du désir, de l'excitabilité...) à ne pas méconnaître.

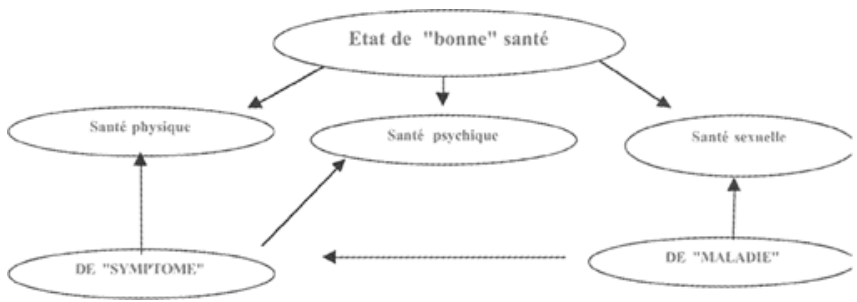

b) le concept "DE symptôme" " désexualise " le trouble Figure 1 : Modélisation du concept DE maladie/DE symptôme [5]. Le dysfonctionnement érectile (DE) est un marqueur pertinent de l'état de la santé sexuelle mais aussi globale (physique, psychique et sexuelle selon la définition de IOMS) et de la qualité de vie de l'homme (et souvent du couple). Ce concept de $D E$ "symptome" et/ou DE "maladie" donne toute sa place au DE dans la prise en charge (primaire ou secondaire) de l'état de santé globale du patient sans aller au-delà de sa demande. 
érectile en ne tenant pas compte de son éventuel retentissement sur la qualité de vie (Figure 1). Le DE n'a alors qu'une valeur de symptôme d'anomalies sous-jacentes médicales ou non, connues ou non, modifiables ou non. II s'apparente à une fenêtre s'ouvrant sur de multiples et fréquentes situations à risque (surpoids, addictions, stress, mauvaise observance...) et/ou sur des pathologies chroniques (athérosclérose, diabète, dépression, iatrogénie...) de mieux en mieux identifiées par l'épidémiologie $[5,20$, $23,24,25,33,34,39]$.

c) le concept de profil de risque de DE se fonde sur l'action plus synergique qu'additive des multiples facteurs intriqués à chaque étape (physiologique, physiopathologique, clinique et thérapeutique). Ainsi, la survenue d'un DE n'est jamais un hasard ce qui impose de rechercher, de principe, des facteurs :

1) prédisposants personnels, biomédicaux, socioculturels et environnementaux,

2) déclenchants et/ou d'entretien (maladies, évènements de vie, conjugopathies...) susceptibles d'expliquer le pourquoi de chez cet homme, de maintenant et de sa persistance $[5,10,20]$. Pour cette raison, l'évaluation du profil de risque est un préalable indispensable à toute prise en charge d'un DE car son objectif est de dépister et/ou prévenir les divers comorbidités et/ou facteurs de risque (en sachant que leur correction est une condition nécessaire mais non toujours suffisante) [5].

Cette distinction DE "symptôme/maladie" associée au concept de profil de risque n'est pas que théorique car elle a plusieurs incidences pratiques $[5,6]$ en permettant :

- une approche individualisée qui replace la sexualité et ses troubles dans leur véritable contexte, c'est-à-dire, d'être une fonction relationnelle essentielle mais complexe car sans normes précises $[7,11,13]$;

- à chaque soignant de savoir jusqu'où il peut et souhaite aller en fonction de ses connaissances et de son intérêt sexo-andrologique ;

- de donner au trouble érectile toute sa place dans une prise en charge globale de la santé du patient sans aller au-delà de la demande du sujet. Si le DE est toujours "symptôme", il est souvent, mais non toujours, "maladie" car la fonction sexuelle est facultative et chacun vit sa sexualité comme il le veut (ou le peut) ;

- de respecter les préceptes d'une médecine humaniste (rôle d'homme aidant un autre homme malade) et non uniquement technicienne ;

- un dépistage opportuniste et proactif des morbidités chroniques et des situations à risque pour la santé (même si le DE n'est pas un problème pour le patient).

Le bilan d'un DE peut (et même doit) s'inscrire dans une politique de prévention de la "non-santé" et de promotion de la "bonne santé". Ces priorités de santé publique actuelles ne passent pas par la multiplication du nombre de spécialistes mais par une prise de conscience et un nouvel état d'esprit afin de mobiliser tout le corps médical et de sensibiliser la population. Pour éviter toute médicalisation excessive du DE, le médecin doit toujours se demander quel est son retentissement psychologique, relationnel et social sur la vie quotidienne du patient (et de la partenaire) $([3,10,11,12,25,33,34,46]$. Pour cela, il doit savoir écouter afin d'identifier les besoins, les attentes et les priorités du couple. Ainsi, la "non-concordance" entre un patient demandeur et un médecin non-demandeur n'a plus lieu d'être car le DE "maladie" en tant que maladie (sexuelle) chronique doit intégrer (comme toute maladie chronique) : 1) une dimension biomédicale mais aussi psychosociale, 2) une décision partagée et appliquée par le médecin et le patient.

\section{LA PRESCRIPTION RAISONNEE ACTUELLE}

Ces nouveaux concepts visent à respecter les exigences d'une démarche scientifique de qualité, c'est-à-dire qui associe harmonieusement: 1) le concept de la Médecine Fondée sur les Preuves (EBM), 2) la réalité d'une pratique et d'une compétence médicale clinique, 3) la preuve d'un service rendu au patient. Ils sont à l'origine des indications raisonnées et pragmatiques actuelles, c'est à dire, toujours orientées par les données de l'étape clinique [6, 12 , $34,35,44]$ mais dont le niveau de preuve scientifique actuel ne relève encore pour l'essentiel, que d'accords forts professionnels et de consensus. Devant tout patient consultant pour une $\mathrm{DE}$, une hiérarchie du bilan peut être établie en fonction :

a) de la qualification des intervenants en distinguant ce qui peut et doit être fait par tout médecin (généraliste ou spécialiste) en premier contact et ce qui relève plus spécifiquement du médecin spécialiste (du DE ou d'un autre domaine) ;

b) des prescriptions de première intention (biologiques, les seules de pratique quotidienne) qui peuvent être prescrites par tous les médecins ou de deuxième ou troisième intention, relevant le plus souvent du spécialiste du $\mathrm{DE}$;

c) des objectifs en différenciant ce qui relève du dépistage d'un problème de santé publique (diabète, facteurs de risque cardiovasculaire, cancer de prostate...) ou du bilan orienté par les données cliniques [6].

Quelle que soit l'étiologie du DE, une investigation complémentaire n'a aucun intérêt si la conduite diagnostique ou thérapeutique n'est pas modifiée. Comme pour tout problème de santé, le médecin doit s'efforcer d'intégrer dans sa prescription pour $\mathrm{DE}$, une démarche de dépistage, de prévention et d'éducation dans ses dimensions biomédicale, sociétale et économique. Finalement, cette prise en charge permettra de sortir du constat bien résumé par Pasini [42], "les psychiatres doivent abandonner la verbalisation dans laquelle ils se sont enfermés et participer avec les thérapies d'accompagnement aux nouvelles solutions pharmacologiques..." tandis qu' "il faut aussi que les médecins urologues, andrologues vérifient quand la prescription 
des nouveaux médicaments est plus utile à eux qu'à leurs patients... pour (traiter) leur impuissance de médecin...".

\section{BILAN DE PREMIERE INTENTION}

\section{A. BILAN BIOLOGIQUE}

Les recommandations de 1999 [4, 15, 34, 44] sont toujours d'actualité avec simplement davantage d'attention pour le dépistage des facteurs de risques cardio-vasculaires, priorité actuelle de santé publique. L'âge, le bilan clinique, les recommandations internationales et celles de l'ANAES servent de fil conducteur.

\section{Facteurs de risque cardio-vasculaire}

\section{a) Rationnel}

Les données épidémiologiques (confirmées par l'expérimentation animale) ont montré qu'ils étaient prédictifs du $D E$ d'où une prévalence et une sévérité accrues du DE en cas de pathologies cardiovasculaires $[5,30,31,33,39$, 47]. Ce n'est pas étonnant car l'érection est avant tout un phénomène vasculaire marqué par le remplissage sanguin des corps érectiles biochimiquement déclenché par la sécrétion endothéliale et neuronale du monoxyde d'azote (NO). Les corps érectiles fonctionnent comme une véritable éponge active [5] ce qui explique que l'érection peut être assimilée à un authentique test d'effort vasculaire ultrasensible. En fait, les corps érectiles sont des "vaisseaux" hyper spécialisés dans la mesure où ces «éponges " sanguines ne fonctionnent que de façon intermittente sous l'action pro ou anti-érectile spécifique du cerveau [5]. Ainsi, tout défaut marqué d'apport artériel, quel que soit son origine, peut provoquer à lui seul un DE à l'exemple de la rare étiologie post-traumatique du sujet jeune.

Mais, la baisse du NO d'origine endothéliale résulte presque toujours d'une intrication de mécanismes physiopathologiques (athérosclérose, dysfonction endothéliale, ischémie chronique) (Figure 2). En aggravant le profil de risque, ils sont responsables d'un mauvais fonctionnement de l'éponge érectile par défaut d'apport artériel et/ou myocontractilité insuffisante et/ou une fibrose des tissus érectiles expliquant un verrouillage veineux défectueux. Exactement comme au niveau de la circulation générale, l'endothélium des corps érectiles joue un rôle physiologique majeur dans l'érection d'où le récent concept Dysfonction Erectile $=$ Dysfonction Endothéliale selon lequel le DE ne serait qu'un symptôme clinique d'une atteinte endothéliale diffuse et peut-être, un signal d'alerte précédant la survenue d'accident vasculaire aiguë $[17,30,31,35,39,47,51]$.

Mais, ce passage du "tout psychogène" des années 1980 au "tout vasculaire" des années 2000 apparaît tout aussi excessif car il ne prend pas suffisamment en compte les autres facteurs étiologiques (biomédicaux, environnementaux, psychologiques) très souvent présents $[5,10,17,20$, $23,33,35,47]$. Néanmoins, sa conséquence pratique est que tout DE doit être considéré, jusqu'à la preuve du contraire, comme un marqueur d'anomalies cardiovas-

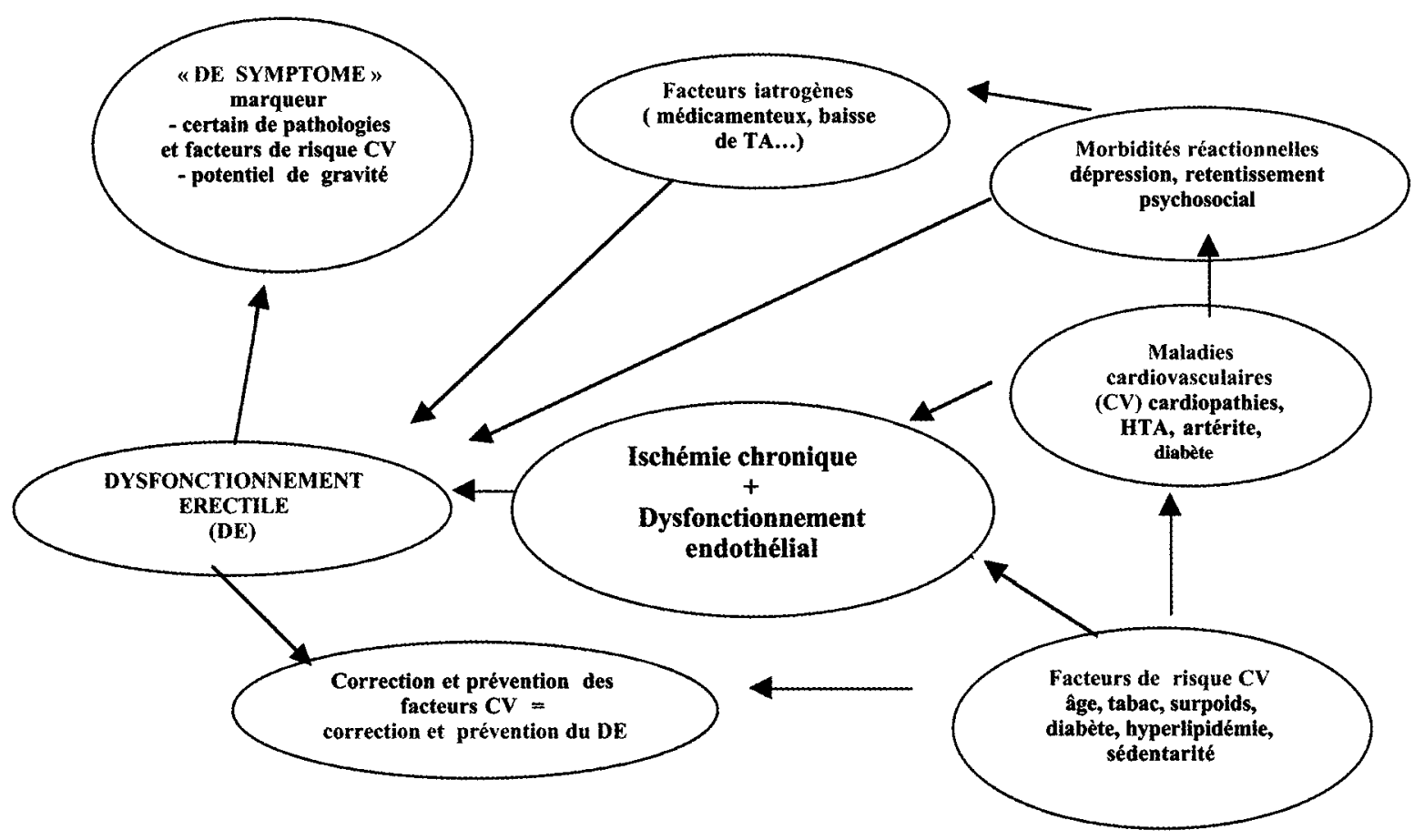

Figure 2 : Schéma des mécanismes physiopathologiques potentiellement impliqués dans le dysfonctionnement érectile (DE) d'origine vasculaire. Ce schéma explique qu'après 35 ans : 1) sa survenue doit être considérée de principe, comme un symptôme marqueur d'une pathologie cardiovasculaire ("DE symptôme "), 2) la recherche systématique des facteurs de risque cardiovasculaires lors de l'évaluation du profil de risque de DE s'intègre dans une démarche de prévention globale des maladies cardio-vasculaires, 3) le caractère délétère du profil de risque [5]. 
culaires (tout particulièrement après 35 ans) $[5,10,35$, 47]. Ce dépistage opportuniste des facteurs de risques et des maladies chroniques cardiovasculaires (connues ou non) permet de répondre à un double impératif :

- thérapeutique : le traitement du DE nécessite dans tous les cas, leur correction (au moins en tant que cofacteur) en incluant une modification à long terme de l'hygiène de vie (ciblée sur la lutte contre la sédentarité, la réduction pondérale, l'arrêt du tabagisme...) et parallèlement, le contrôle pharmacologique des facteurs de risque individuels (HTA, diabète, dyslipidémie, stress...).

- préventif : quoique les "tueurs" cardio-vasculaires (et d'érection) soient bien connus (tabac, cholestérol, sédentarité, surpoids, antécédents familiaux), les populations exposées ne font pas l'objet d'un dépistage systématique en France. Pourtant, la prévention de l'athérosclérose est un enjeu majeur actuel de santé publique afin d'éviter les accidents d'athérothrombose cardio-vasculaire (première cause de mortalité et troisième cause de décès prématuré) qui sont les témoins cliniques du processus évolutif de cette maladie chronique redoutable. Son évolution souvent silencieuse et progressive sans symptôme avant coureurs $[28,30,41]$ souligne tout l'intérêt du DE symptôme afin de détecter au plus tôt des anomalies chez les personnes à risque d'autant plus que leur nombre augmente. L'OMS vient de déclarer le récent "syndrome métabolique " (qui s'intègre dans la pandémie actuelle de "diabésité") problème majeur de santé car il concernerait près de $30 \%$ des hommes européens $>\mathbf{5 0}$ ans et multiplierait au moins par trois le risque cardio-vasculaire $[28,41]$. Comme pour le profil de risque de $D E$, c'est plus l'association des anomalies que leur niveau qui constitue un danger.

Véritable tournant, ces progrès physiopathologiques ouvrent la perspective d'un traitement « préventif » du DE dans la mesure où : a) le DE d'un certain nombre d'hommes de moins de 40 ans jusqu'à présent classé "psychogène" reflète probablement la présence de lésions vasculaires sous-jacentes méconnues (d'où l'importance de toujours préciser les antécédents cardio-vasculaires familiaux et personnels), b) les sujets ayant une hypertonie orthosympathique semblent les plus exposés notamment en cas de tabagisme, de stress, d'hyperanxiété et de surmenage $[5,20]$.

Remarque : selon la définition de l'OMS [28, 41], le "syndrome métabolique " associe au moins trois des cinq anomalies suivantes: a) cliniques (surcharge pondérale avec un tour de taille $>102 \mathrm{~cm}$, hypertension artérielle), b) biologiques : glycémie $>110 \mathrm{mg} / \mathrm{dl}$, triglycérides $>150 \mathrm{mg} / \mathrm{dl}$, cholestérol $H D L<40 \mathrm{mg} / \mathrm{dl}$ [41]. Un tour de taille $>90 \mathrm{~cm}$ et un taux élevé de triglycérides identifieraient de façon simple, $80 \%$ des sujets ayant un syndrome métabolique.

\section{b) Indications et modalités}

Ce bilan guidé par la clinique concerne en priorité l'homme après 35 ans et s'intègre dans la prise en charge du syndrome métabolique $[5,23,28,30,34,41$, 44]. En France, les modalités de dépistage et de diagnostic biologique des divers facteurs de risque cardio-vasculaires font l'objet de recommandations de l'ANAES avec : a) une glycémie à jeun après 40 ans et/ou en cas d'obésité à tendance abdominale et/ou d'antécédents familiaux de diabète non insulinodépendant, b) un bilan lipidique (profil lipidique) chez les sujets à risque cardio-vasculaire et/ou avec des antécédents familiaux de troubles lipidiques et/ou de prise de poids $[28,47]$. Un bilan de dépistage (cholestérol et triglycéride) peut être indiqué chez tout sujet à partir de 2030 ans n'en ayant pas eu depuis 5 ans (avant 50 ans) ou 3 ans (après 50 ans), c) un dosage du CRP, marqueur inflammatoire et facteur prédictif potentiel du risque d'accidents cardio-vasculaires aigus et du syndrome métabolique [41]

\section{Hormonal}

\section{a) Rationnel}

Dominé par l'hypogonadisme et, dans une moindre mesure, l'hyperprolactinémie, sa place réelle reste controversée car le bilan hormonal systématique chez les patients tout venant consultant pour DE s'avère peu "rentable" avec moins de $10 \%$ d'hypotestostéronémies et moins de $1 \%$ d'hyperprolactinémies $[5,9,15,23,33]$. En fait, l'hypogonadisme entraîne souvent, mais non obligatoirement, un DE (les effets de la testostérone sur la sexualité concernant plus le désir et la trophicité des organes génitaux que l'érection elle-même). L'hypogonadisme complet est peu fréquent à la différence de l'hypogonadisme partiel, entité encore imprécise et controversée notamment dans ses liens avec le vieillissement [16, 32, 33].

Le déficit en androgènes lié à l'âge (DALA ou "andropause") est une réalité qui concerne près de $20 \%$ des hommes après 50 ans mais avec des variations interindividuelles considérables. Ses signes cliniques sont peu spécifiques, communs à de multiples morbidités et très variables dans le temps. Par exemple, l'hypothyroïdie patente ou partielle, très fréquente chez les sujets âgés a des signes cliniques (fatigue, asthénie, dépression) proches du DALA. Par ailleurs, il n'y a pas de parallélisme étroit entre la sévérité de la $D E$ et la baisse de testostérone $[9,10,32]$. L'activité sexuelle ne semble pas nécessiter beaucoup de testostérone et son seuil d'action varierait selon les organes cibles (et probablement les individus). De surcroit, une stimulation sexuelle intense peut compenser un manque de testostérone (notamment chez le sujet âgé) et la baisse de testostéronémie peut n'être que transitoire et multifactorielle (pathologies aiguës, stress, dépression, iatrogène et... baisse de l'activité sexuelle) [32].

Malgré ces réserves, un déficit en androgènes lié à l'âge (DALA ou "andropause") [16, 32, 34, 35, 44] doit être recherché de principe pour des raisons, a) épidémiologiques : la majorité des patients ayant un DE et/ou une "andropause" ont plus de 50 ans [19, 23, 33], b) physiopathologiques : l'hypogonadisme peut être la cause ou la conséquence du $D E, c)$ cliniques : le $D E$ est un des tout premiers motifs de consultation révélateurs d'un DALA, d) thérapeutiques : si l'androgénothérapie permet souvent le 
retour d'une érection naturelle, elle corrige aussi et surtout les autres conséquences du déficit androgène, qu'elles soient sexuelles (se sentir mieux et améliorer le désir et la satisfaction sexuelle qui sont des paramètres importants dans les mécanismes du DE) [16] ou non sexuelles (réduction de certains facteurs de risque cardiovasculaire, mieuxêtre global...).

Dans tous les cas, la confirmation de l'étiologie hormonale du DE exige la conjonction d'anomalies des dosages hormonaux et de signes cliniques d'autant plus que ces derniers sont souvent peu spécifiques.

\section{b) Indications et modalités}

Devant un homme consultant pour DE, le consensus actuel est de ne doser la testostéronémie : a) après 50 ans de principe, dans le cadre du dépistage d'une "andropause" favorisant le DE (sauf si les érections nocturnes et le désir androgénodépendants sont présents), b) quel que soit l'âge, en cas d'anomalie cliniques (baisse du désir, signes d'hypogonadisme) $[9,16,35,44]$.

La prolactinémie d'emblée n'est justifiée qu'en cas de suspicion clinique (gynécomastie, iatrogène, baisse certaine du désir) en sachant qu'une hyperprolactinémie s'accompagne presque toujours d'une hypotestostéronémie [ 9 , 44]. L'intérêt du dosage de la DHEA n'est toujours pas prouvé actuellement. La variabilité inter et intra-individuelle de ces dosages implique que toute anomalie franche ou à fortiori, limite, doit être contrôlée en s'assurant de bonnes conditions techniques [32].

Si le dosage de la testostéronémie totale peut suffire en première intention, la testostéronémie biodisponible (multiple de la testostérone libre) est préférable en lui associant si besoin, un dosage de $\mathrm{LH}$, de $\mathrm{FSH}$ et de prolactine selon les recommandations récentes de l'International Society for the Study of the Ageing male (ISSAM) [16]. En pratique, une testostéronémie biodisponible supérieure à la valeur normale de l'homme jeune élimine un déficit androgénique [32]. En cas de baisse confirmée, une augmentation de LH orientera vers un traitement androgène, une baisse de LH nécessite un bilan endocrinien, notamment hypothalomohypophysaire.

\section{Urologique}

\section{a) Rationnel}

La place de l'urologue est particulière car il est : a) le référent naturel du " versant organique " du DE en cas d'échec de la première prise en charge et/ou de pathologies génitales impliquées dans les troubles sexuels (courbures de verge acquises ou non, séquelles de priapisme et de traumatisme, micropénis, anomalies du prépuce...) [5, 34], b) directement à l'origine d'un nombre non négligeable de DE iatrogènes. Tous les traitements des cancers génito-urinaires pelviens (qui représentent actuellement la moitié des cancers de l'homme) ont des effets délétères sur les fonctions sexuelles d'où le souci actuel de développer des méthodes visant à préserver/récupérer la sexualité $[14,36$, 40].

De façon plus inattendue, les troubles obstructifs sympto- matiques du bas appareil urinaire (TUBA) essentiellement provoqués par l'hyperplasie bénigne de la prostate (soit plus du tiers des hommes après 50 ans) sont apparus comme un facteur de risque indépendant de DE $[5,38]$. Ses principaux mécanismes semblent plus indirects que directs associant diversement : a) les effets secondaires de certains médicaments, b) l'altération de la qualité de vie (troubles du sommeil et fatigue) du patient et de sa partenaire parallèlement à la sévérité des troubles urinaires qui retentit logiquement sur leur vie sexuelle $[38,45]$, c) la mauvaise réputation de « la prostate » bien ancrée dans l'imaginaire de beaucoup d'homme quant au risque de perte de "virilité" liée à ses traitements et surtout, beaucoup plus justifié, de cancer (premier cancer de l'homme en 2000).

\section{b) Indications et modalités}

Un dosage de PSA (antigène spécifique de la prostate) totale est actuellement indiqué [14] chez tous les hommes de plus de 50 ans, qu'il y ait ou non un DE : a) dans le cadre du dépistage individuel du cancer de prostate (jusqu'à 75 ans) ou à partir de 45 ans en cas de terrain à risque (antécédent familial de cancer de prostate, origine africaine ou antillaise) (recommandations de l'Association française d'Urologie 2003), b) si une androgénothérapie est envisagée, c) à fortiori en présence d'anomalies cliniques (toucher rectal, troubles mictionnels) souvent présentes. Le dosage de créatininémie n'est pas systématique mais recommandé après 50 ans ou avant en cas de comorbidités source de néphropathies (diabète, pathologie et/ou facteurs de risques cardio-vasculaires...) souvent présentes imposant alors le calcul de la clearance [28].

\section{B. AVIS SPECIALISES COMPLEMENTAIRES}

Leur demande peut être liée à la mise en évidence d'une pathologie associée ou aux limites de compétence du premier soignant $[6,12,34,44,56]$. Ils peuvent être spécifiques au trouble sexuel (notamment en cas de facteurs de gravité) et/ou à la pathologie supposée responsable du DE et/ou dans le cadre de la prise en charge globale de la santé du patient. En pratique, les plus demandés sont à visée cardiologique et sexo-psychologique, conséquence logique des prétendus "dangers cardio-vasculaires" des inhibiteurs de la PDE 5 et des lacunes de formation sur la santé sexuelle. Selon les cas, le recours à d'autres avis complémentaires (urologique, neurologique, psychiatrique, endocrinologique...) peut être nécessaire.

\section{Sexo-psychologique}

\section{a) Rationnel}

La sexualité est une fonction importante mais très particulière car non vitale, facultative, sans normes précises et en règle vécue à deux avec d'importantes variations inter mais aussi intra individuelles. Cette diversité contraste avec les « modèles " sociétaux actuels qui cultivent la compétition et les indicateurs de performance, expliquant une perte de repères chez nombre de personne. Outre la procréation et l'érotisme, la sexualité a aussi et d'abord un rôle identitaire puis relationnel trop souvent 
négligé quoique essentiel. Enfin, le $D E$ est très souvent intriqué à d'autres troubles sexuels et it n'est pas rare qu'il ne soit qu'une complication plus " démonstrative » d'une dysfonction sexuelle (éjaculation rapide, troubles du désir, de l'excitabilité...) sous-jacente.

Cette complexité de la sexualité s'explique par le fait que "chacun fait l'amour avec son sexe mais aussi avec son corps, son imaginaire, son inconscient et un partenaire qui est le plus souvent, de sexe et de mode d'emploi différent, le tout dans un environnement rempli de fausses croyances et de mythes sous-tendus par les nouvelles normes sociales d'épanouissement sexuel obligatoire" [11]. Le jeu et l'échange amoureux ou érotique, la sensualité, le savoirfaire sexuel dépendent ainsi d'éléments cognitifs multiples car le sexe n'est pas inné. En fail, il s'apprend (Figure 3) au travers du "formatage" de l'enfance, des expériences sexuelles, de l'environnement socioculturel et de(s) partenaire(s). Ainsi, pour comprendre le symptôme sexuel et la sexualité d'un individu, l'intimité du couple (qu'il s'agisse de la qualité de leur relation et/ou de la complémentarité de leurs capacités sexuelles) doit toujours être prise en compte.

Toute survenue d'un DE peut fragiliser l'homme (et sa partenaire) en raison de réactions émotionnelles et psychologiques négatives ("il n'est plus un homme", "je ne l'attire plus"). Des cercles vicieux surviennent fréquemment, résultats de frustrations, d'ignorance et de réactions inadaptées tant chez l'homme (conduite habituelle d'évitement, angoisse anticipative d'échec) que chez sa partenaire (frustration, culpabilité, ressentiment, crainte d'une relation extraconjugale...) $[3,7,8,10,13,46]$. Ces pertes d'intimité et de complicité sont d'autant plus pénalisantes qu'il s'agit souvent d'hommes déjà fragilisés par leur statut de malade chronique (cardiaques, diabétiques, hypertendu...) $[5,10]$.

Le DE peut être ainsi la cause ou la conséquence de dépression ou d'anxiété généralisée. Dans les deux cas, ces perturbations sont aussi des marqueurs de la souffran-

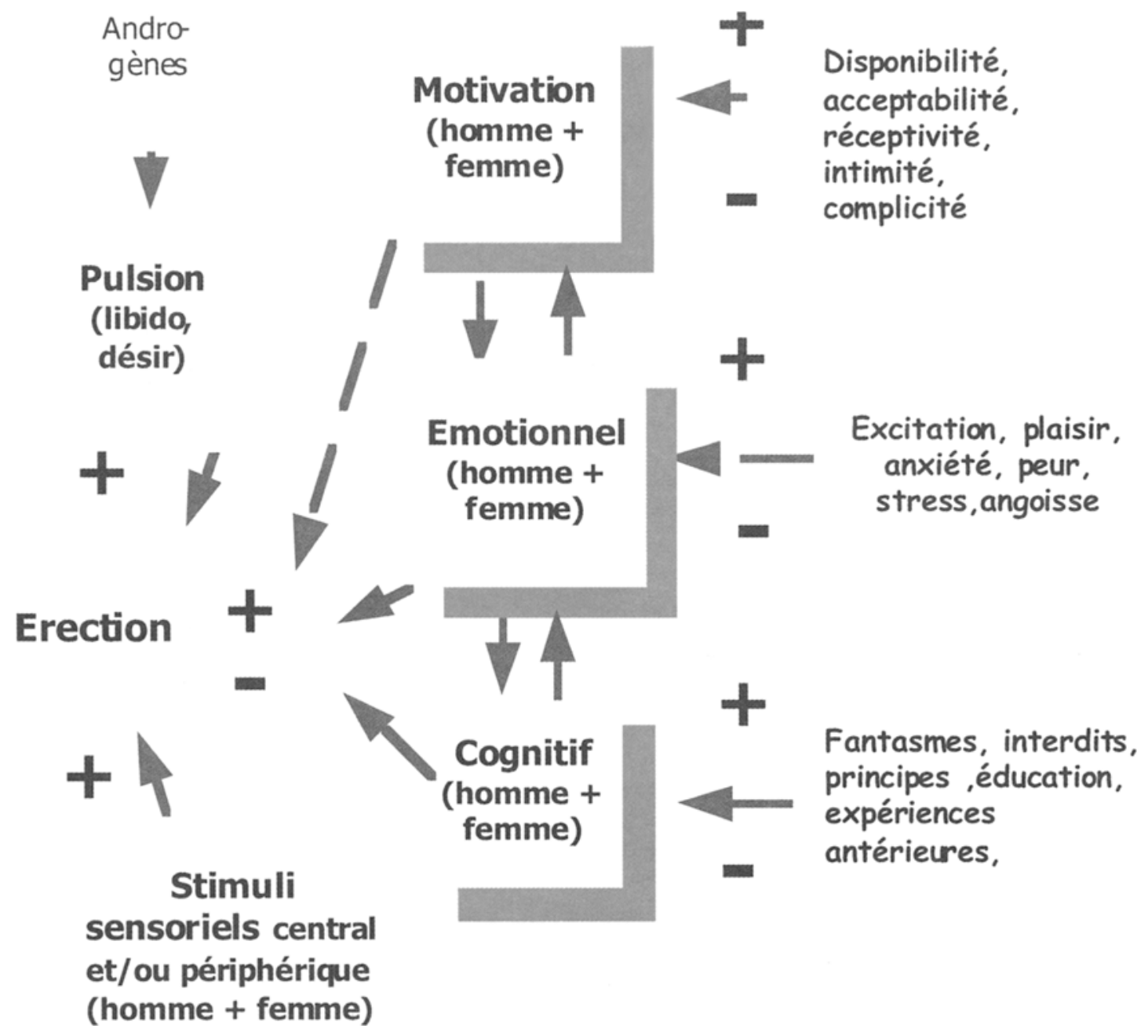

Figure 3 : Multiplicité des paramètres susceptibles de moduler le déclenchement ou non, d'une érection en réponse à des stimulations centrales et/ou périphériques. Ce rôle pro et antiérectile du cerveau, premier organe sexuel de l'homme (et de la femme), explique la nécessité de toujours prendre compte le "contexte sexuel érectile ", notamment la présence ou non d'un désir et d'une motivation sexuelle, le contexte émotionnel et les antécédents sexuels du patient, mais aussi celui de la partenaire et du couple [5]. 
ce du patient ce qui laisserait supposer que les patients qui consultent actuellement sont ceux qui sont les plus en détresse. En l'absence de communication ou de demande d'aide, ce trouble relationnel risque de s'exacerber et même de se pérenniser quoique le(s) facteur(s) déclenchant(s) aient disparu. Le DE peut provoquer tant chez l'homme que la femme, une remise en cause déstabilisante d'autant plus qu'il peut révéler ou aggraver une pathologie de couple ("conjugopathie" ou désynchronisation sexuelle) sous-jacente d'où l'importance de savoir si la partenaire : a) est proactive, réactive ou antiactive, b) souffre ou non du $D E, c)$ a ou non un trouble sexuel $[8,10$, 11]. Ces dangers potentiels expliquent:

- l'intérêt d'une prise en charge précoce et la plus grande difficulté à traiter un DE ancien ;

- les objectifs des sexothérapies qui visent à corriger les fausses idées, stopper les cercles vicieux de l'anxiété, déculpabiliser, aider à comprendre le fonctionnement psychologique et physique de l'autre, améliorer la communication tout en respectant l'altérité et la liberté, retrouver la confiance en soi et en l'autre car le couple est la dimension vraie de la sexologie [7] ;

- l'intérêt d'une prise en charge d'inspiration "sexothérapique" de principe pour une prise en charge réellement globale et pour accompagner la prescription [3, 8, 11, $34,44,56]$. Néanmoins, si les signes anxiodépressifs face à la maladie et au changement font partie des réactions normales d'adaptation, un des secrets de la sexualité (et de la vie) est de savoir s'adapter. Cela explique qu'en pratique, le retentissement du DE varie considérablement en fonction de multiples paramètres, propres ou indépendants, du patient : personnalité, environnement (attitude du partenaire, événements de vie), capacités d'adaptation et de résilience, hiérarchies de valeur...

Remarque : la recherche d'une dépression réactionnelle ou plus grave est facilitée par l'emploi d'une échelle de dépression simple à deux questions : au cours du mois écoulé, vous êtes-vous senti abattu, déprimé, désespéré ? au cours du mois écoulé, avez-vous souvent ressenti une baisse d'intérêt ou de plaisir dans votre vie de tous les jours [34]?

\section{b) Indications et modalités}

A la différence de la psychanalyse qui explore le domaine de l'inconscient pour faire prendre conscience des conflits sous-jacents, les psychothérapies cherchent à corriger les symptômes en travaillant diversement au niveau du réel, de l'imaginaire et du symbolique. Les psychothérapies joueront plutôt sur les blocages inconscients qu'il s'agisse d'interdits, de principes d'ordre éducatif, culturel, religieux... Les sexothérapies relanceront la dynamique de couple, notamment la communication souvent bloquée à tous ses niveaux (sexuels ou non). En jouant sur le désir provoqué ou l' "envie d'avoir envie", elles cherchent à modifier les "tue désirs" et les "érosions du désir " que sont la routine, l'absence d'intimité et de complicité, les problèmes de communication dans le couple $[3,7,8,11]$.

Les sexo et les psychothérapies ont des indications spécifiques selon l'origine primaire ou secondaire du trouble érectile (événements de vie, manque de culture érotique, excès d'émotivité, d'anxiété, de timidité, de narcissisme, de perfectionnisme). Afin d'améliorer leur efficacité, les sexothérapies peuvent combiner les approches comportementales et cognitives, les thérapies de couples, les techniques de relaxations, les conseils pratiques et les médicaments. En pratique, la sexologie n'étant qu'une compétence complémentaire à une formation initiale (psychiatrique, psychanalytique, psychologique ou autre), le choix du traitement doit être toujours adapté au patient (et idéalement adoptée de façon consciente et complice par les deux partenaires). La prise en charge d'inspiration sexologique [8] mérite une place à part car cette simple psychothérapie de soutien, réalisable par tout soignant, suffit souvent à rassurer, à déculpabiliser le patient et/ou la partenaire et à "relancer" leur sexualité. Elle consiste en une information éclairée sur la sexualité et ses troubles via des explications, une dédramatisation, une réassurance et des conseils de bon sens. Elle fait partie intégrante de l' " AMES » (Aide Médicalisée à l'Erection et à la Sexualité" par analogie avec l'Aide Médicale à la Procréation), prise en charge habituelle de première intention qui associe une prise en charge d'inspiration sexologique, une prescription bien accompagnée de traitements pharmacologiques (spécifiques ou non), et la correction des facteurs de risque de DE $[6,8,11,44,56]$.

A un stade de plus, l'emploi de thérapies cognitivo-comportementales est indiqué. Ces thérapies centrées sur la guérison du symptôme visent à agir sur les pensées, les croyances et les comportements souvent erronés (culpabilité, conduite d'évitement, repli sur soi...) sur lui-même ou sur son entourage. Leur but est de redonner au symptôme $D E$, son véritable sens (manque de confiance, d'affirmation et d'estime de soi). D'autres méthodes (relaxation, sophrologie, hypnothérapie...) peuvent être également utilisées pour redonner une confiance en soi, réduire l'anxiété et retrouver une relation avec son corps. En cas de "conjugopathie" ou de désynchronisation sexuelle, les thérapies de couple cherchent à (re)créer une relation et une dynamique entre les partenaires. La prise de conscience et la correction des résistances passent notamment par une meilleure communication pour que chacun entende et comprenne l'autre dans ses difficultés et son altérité [11]. Le rôle du sexologue consiste à atténuer les souffrances et les dysfonctions sexuelles, et, s'il le peut, à les guérir. En apprenant au sujet à comprendre et à maîtriser ses émotions souvent négatives, le savoir-faire du sexothérapeute facilitera la récupération d'un savoir-faire sexuel trop souvent oublié ou mal compris par le patient et le couple du fait des cercles vicieux déstructurants de l'anticipation négative en s'aidant, si besoin, d'inhibiteurs de la PDE 5 pour minimiser la programmation du désir. Quoique critiqués actuellement pour un défaut d'information et de validation [29], ces traitements aident à mieux supporter le $D E$, même s'ils sont souvent moins efficaces en cas d'ano- 
malies organiques avérées. Le recours à un sexologue, un psychologue ou un psychiatre (selon la gravité apparente des troubles sexopsychologiques) $[3,8,11,44,46]$ est :

\section{recommandé :}

- d'emblée en cas de DE "maladie" sévère (fondée sur une souffrance et une altération de la qualité de vie à ne pas confondre avec un trouble psychiatrique nécessitant un avis spécifique) associée ou non à des signes de gravité liés au patient (troubles sexuels primaires ou multiples, DE d'évolution supérieure à deux ans, antécédents de traumatismes ou de violences sexuelles, de maladies et de traitements psychiatriques, sujet jeune, facteurs de risques psychologiques) ou à son environnement (évènements de vie plus ou moins récents qu'ils soient professionnels, veuvage, divorce difficile...), ou en cas de difficultés relationnelles (notamment conjugopathies) manifestes lors de la première consultation. Le manque de motivation et/ou une très mauvaise entente du couple sont ainsi des facteurs majeurs d'échec ;

- secondairement en cas d'échec du premier traitement (autre que l'intolérance d'un médicament). II convient alors de rechercher une inadaptation de sa vie sexuelle liée à un manque de stimulation érogène ou à des difficultés relationnelles. La distinction entre cas simple ou complexe lors du premier bilan vise à minimiser le risque d'échec. Un des rôles du suivi est ainsi d'amener progressivement le patient à mieux harmoniser ses attentes et ses souhaits avec la réalité en comprenant qu'il doit passer à un autre niveau d'analyse que le souci de sa seule "performance" érectile sans se préoccuper des aspects relationnels;

Indispensable d'emblée avant toute prescription médicamenteuse en cas de trouble psychiatrique manifeste ou de doute sur l'aptitude "psychique" (crainte d'une éventuelle dangerosité type déviance sexuelle notamment une perversion).

\section{Cardio-vasculaire}

\section{a) Rationnel}

L'importance anatomophysiologique de la composante vasculaire, explique les trois objectifs de son bilan [17, $30,31]: 1)$ dépister et, si besoin, prendre en charge d'éventuelles pathologies/facteurs de risque cardio-vasculaires (DE "symptôme"), 2) vérifier l'aptitude physique à la poursuite ou la reprise d'un rapport sexuel qui correspond (habituellement) à un exercice physique modéré de l'ordre de montrer deux étages à pied un peu rapidement, c) vérifier l'absence d'interactions médicamenteuses avec les inhibiteurs de la phosphodiestérase.

En pratique quotidienne, une évaluation cardiologique minimale est indispensable car le sujet jeune, non sédentaire et en apparente bonne santé qui consulte pour DE reste l'exception. La population "cardiologique type" a un profil particulier lié à : a) son âge commun avec la population ayant un $\mathrm{DE}$, les coronariens ayant majoritairement plus de 50 ans, b) son risque spécifique d'accidents vascu- laires aigus liés à la pathologie cardiovasculaire et/ou à une mauvaise observance par effet iatrogène plus supposé que réel et/ou à la prise de médicaments contre-indiqués (risque d'hypotension artérielle sévère), c) la crainte injustifiée mais toujours présente du soit disant danger des inhibiteurs de la PDE5 [2, 6]. Pourtant, comme les autres, les sujets coronariens ont une demande légitime de qualité de vie sexuelle et globale.

\section{b) Indications et modalités}

Quel que soit son âge, tout homme consultant pour DE doit bénéficier d'une évaluation minimale de son statut cardio-vasculaire (Figure 4) réalisable sans difficultés par tout médecin non-cardiologue.

En 2000, la conférence de Princeton a établi un algorithme de prise en charge des patients ayant une maladie cardiovasculaire [17] en distinguant les patients à risque : a) faible (Figure 5) sans risque significatif à avoir ou poursuivre une activité sexuelle, b) intermédiaire/indéterminée (Figure 6) et élevé (Figure 7) qui nécessitent une évaluation cardio-vasculaire et une contre-indication temporaire de faire des efforts physiques relativement importants. Un avis cardiologique et un éventuel test d'effort sont en règle nécessaires. En pratique, la présence d'au moins trois facteurs de risque peut faire demander un avis cardiologique à l'exception du diabète qui a un risque individuel élevé d'accidents coronariens, d'autant plus que le diabète est ancien et mal suivi. Ainsi, toute survenue d'un DE chez un diabétique (notamment non insulinodépendant) doit faire rechercher une ischémie coronarienne silencieuse car le DE est par lui-même, un signe de gravité témoin d'une complication.

En pratique quotidienne, deux situations doivent être distinguées : 1) le sujet déjà connu et suivi régulièrement qui nécessite une réévaluation (de sa pathologie et de son traitement) et la vérification d'une information bien comprise par son médecin de famille et son cardiologue, 2) le sujet à risque non ou mal suivi chez lequel le dépistage et la prévention des accidents cardio-vasculaires (d'évolution souvent silencieuse et progressive sans symptôme avant coureurs notamment en cas de diabète) sont un enjeu majeur. Le sujet sédentaire et/ou ayant plus de trois facteurs de risque cardiovasculaire apparaît comme le plus en danger et nécessite préalablement un avis cardiologique et une épreuve d'effort. Quoique la majorité des patients se situe dans le groupe à faible risque, ces mises en garde sont des données de la médecine fondée sur les preuves et... d'AMM d'où la nécessité de les respecter et donc de les rechercher d'un point de vue éthique, scientifique et médico-légal (même si les seules vraies contre-indications sont médicamenteuses, l'infarctus du myocarde récent et I'hypotension artérielle < 90/50) [48].

Etant donné la réputation injustifiée du soit-disant " danger » des inhibiteurs de la PDE5, le patient doit être clairement informé que :

- leur tolérance cardiovasculaire est excellente, le test d'effort ayant montré chez le coronarien stabilisé l'ab- 


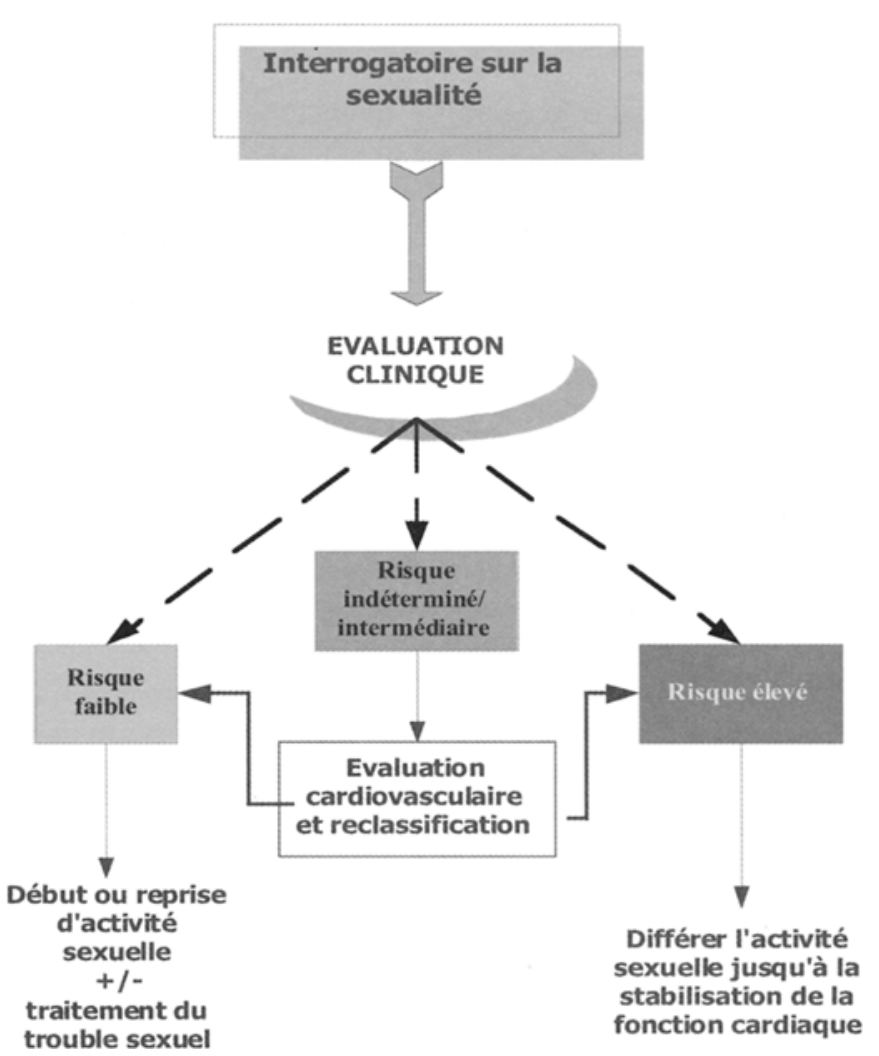

Figure 4 : Algorithme de prise en charge des patients ayant une maladie cardiovasculaire, selon leur risque cardiaque lors d'une activité sexuelle (adapté d'après les recommandations de Princeton) [17].

\section{Risque indéterminé/intermédiaire}

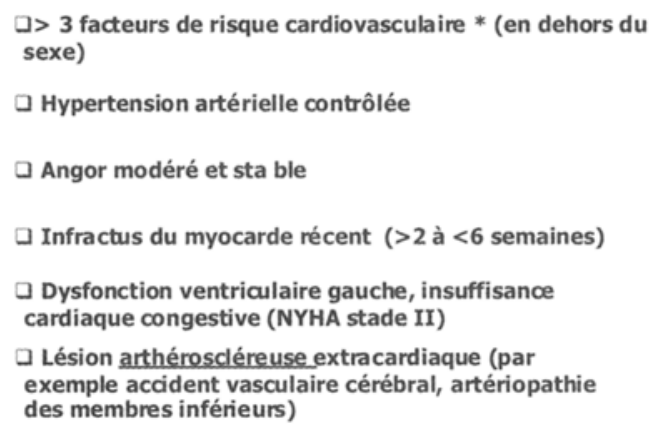

]ypertension artérielle contrôlée

Angor modéré et sta ble

Infractus du myocarde récent ( $>2$ à $<6$ semaines)

Dysfonction ventrialaire gauche, insuffisance cardiaque congestive (NYHA stade II)

\ Lésion ârthéroscléreuse extracardiaque (par exemple accident vasculaire cérébral, artériopathie des membres inférieurs)

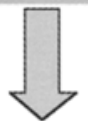

Chez ces patients, une évaluation complémentaire** est nécessaire pour reclassifier dans le groupe à risque faible ou dans celui à risque élevé

Figure 6 : Critères d'évaluation des patients à risque cardlaque indéterminé ou intermédiaire adapté d'après les recommandations du Consensus de Princeton [17].

\section{Risque faible}

Patients asymptomatiques, < à 3 facteurs de risques cardiovasculaire (en dehors du sexe)

a Hypertension artérielle contrôlée

angor léger et sta ble

Revascularisation coronarienne réussie

Antécédent d'infractus du myocarde non compliqué ( $>6-8$ semaines)

a Pathologie valvulaire légère

Dysfonction ventriculaire gauche, insuffisance cardiaque congestive (NYHA stade I)

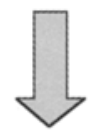

Chez ces patients, aucun test ou évaluation cardiaque spécifique n'est requis avant de débuter ou de reprendre une activité sexuelle et/ou recevoir un traitement pour leurs troubles sexuels

Figure 5 : Critères d'évaluation des patients à risque cardiaque faible adapté d'après les recommandations du Consensus de Princeton [17].

\section{Patient à risque cardiaque élevé}
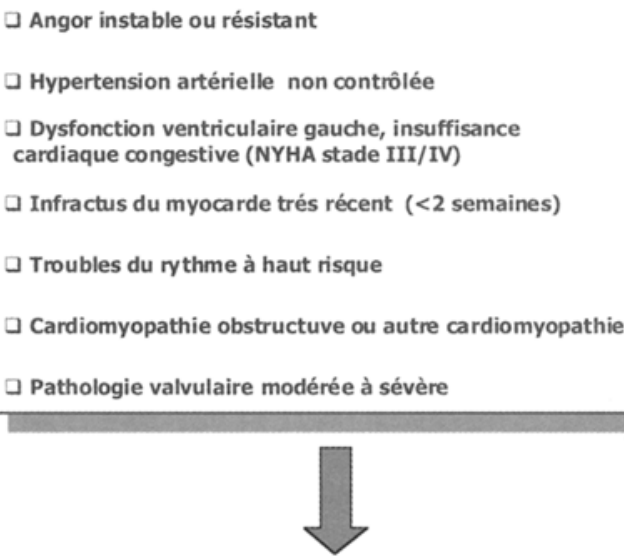

Chez ces patients, l'activité sexuelle doit être différée jusqu'à la stabilisation de l'état cardiaque

Figure 7 : Critères d'évaluation des patients à risque cardiaque élevé adapté d'après les recommandations du Consensus de Princeton [17]. 
sence de danger et plutôt un effet bénéfique, reflet logique de l'effet vasodilatateur coronarien modeste [2, 48] ;

- la possibilité de modifier le traitement cardiovasculaire dans la majorité des cas lors de contre-indication médicamenteuse (dérivés nitrés et donneurs d'oxyde nitrique) sous réserve de l'accord cardiologique (sinon la prise de donneurs de $\mathrm{NO}$ et de dérivés nitrés n'est que temporairement contre-indiquée soit 24 heures avec le sildénafil et le vardénafil soit 48 heures avec le tadalafil);

- en cas de crise angineuse, la résolution spontanée nécessitera un avis cardiologique sinon l'hospitalisation est obligatoire, le patient devant alors signaler la prise éventuelle d'inhibiteurs de PDE 5.

\section{BILAN DE DEUXIEME INTENTION}

Il ne relève à priori que du spécialiste du DE car il n'est indiqué qu'en cas d'échec de la première prise en charge (Figure 8) [6, 18, 22, 43, 44]. Après s'être assuré que la prise en charge initiale a été suffisante ou bien suivie et que des facteurs favorisants n'ont pas été méconnus ou sont apparus lors du suivi, l'injection intracaverneuse (IIC) de drogues érectogènes est actuellement le principal test de deuxième intention (parfois couplé à un examen échodoppler pulsé) [18, 21, 22, 35, 43]. La facilité et le faible coût de ce pharmacotest en font le véritable examen paraclinique qu'il était avant l'arrivée du Viagra $\circledast[1,55]$. II teste la réactivité des tissus érectiles à une puissante stimulation pharmacologique qui varie de 5 à $20 \mu \mathrm{g}$ de prostaglandine $E 1$ selon les données cliniques $[22,55]$. Schématiquement, la réponse est dite :

- positive en cas de survenue d'une érection rigide, c'est à dire sans possibilité de plier la verge, dans les 10 minutes et perdurant 30 minutes. Ces critères retenus par l'European Association of Urology [22] éliminent l'existence de lésions vasculo-tissulaires sévères et orientent en priorité vers une étiologie neurogène ou psychogène;

- négative en cas d'érection non ou peu rigide malgré plusieurs essais à doses croissantes orientant vers des anomalies organiques vasculaires et/ou tissulaires sévères.

Néanmoins, l'existence de faux négatifs (rôle du stress) et de faux positifs (étiologie neurologique ou hormonale par exemple) minimise sa spécificité et sa sensibilité $[4,18,21$, $35,55]$. Ce test diagnostique doit donc toujours être corrélé aux données de la clinique. Pour toutes ces raisons, ce pharmacotest est à réserver aux médecins familiarisés avec la DE et au traitement du priapisme. En pratique quo-

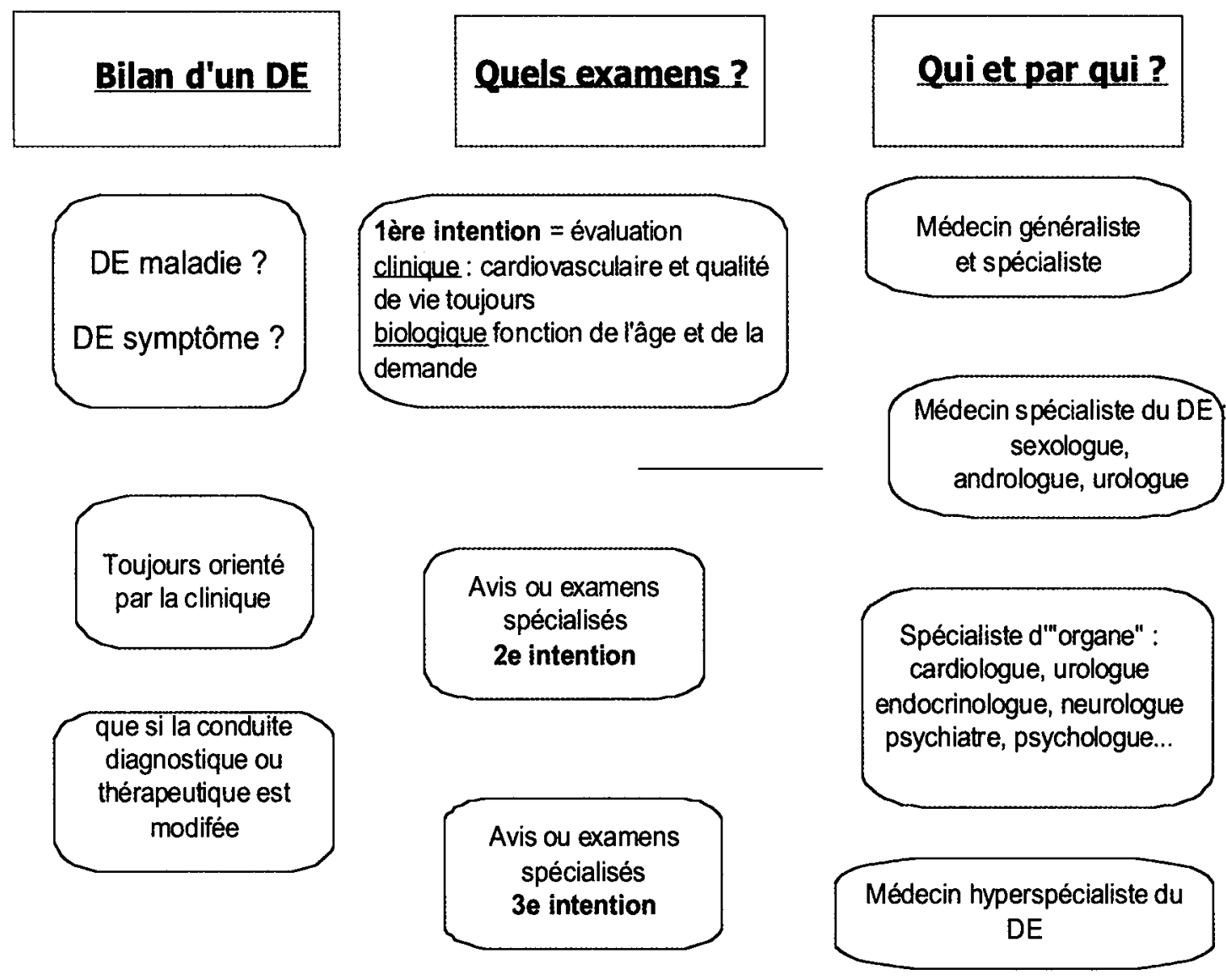

Figure 8 : Synthèse et hiérarchisation du bilan complémentaire d'un dysfonctionnement érectile (DE) en fonction de la qualification du premier soignant [5]. 
tidienne, sa place n'est pas négligeable puisqu'il est encore utilisé comme test diagnostique par près d'un quart des urologues nord-américains et français [42, Enquête PISTES 2002]. Etant donné la valeur diagnostique et probablement pronostique cardiovasculaire du DE, un examen échodoppler pulsé pénien peut être raisonnablement indiqué en deuxième intention chez le sujet présentant des facteurs de risque et/ou des pathologies cardio-vasculaires, à la triple condition : 1) d'être couplé au pharmacotest intracaverneux, 2) d'explorer les gros vaisseaux périphériques, 3) d'être fait par un radiologue et/ou un angiologue référent. Dans tous les cas, a) il faut s'assurer de la disparition de l'érection avant d'autoriser le départ pour éviter le risque de priapisme, b) le constat d'anomalies artérielles caverneuses à l'échodoppler doit évoquer une coronaropathie associée et proposer, si besoin, un test d'effort cardiaque.

\section{BILAN DE TROISIEME INTENTION}

II ne concerne que des explorations hyper spécialisées de l'organe effecteur et/ou de ses commandes. Pour leur majorité, ces tests sont invasifs, chronophages, coûteux, et d'interprétation difficile par manque de données chez des sujets sains, fiabilité insuffisante et reproductibilité aléatoire car trop opérateur dépendant $[1,4,15,18,35,37,38,44$, 53]. En pratique peu prescrits, leurs indications et leurs réalisations relèvent strictement de l'hyperspécialiste du DE $[18,22,44,53]$. Deux enquêtes récentes illustrent bien l'évolution des pratiques. Un échantillon représentatif de 507 urologues américains [43] s'occupant quasiment tous de DE montrent que $80 \%$ utilisent le test pharmacologique oral par sildénafil avant de demander un test diagnostique, d'où la baisse des investigations paracliniques (rigidimétrie nocturne : $13 \%$, échodoppler: $10 \%$, cavernométrie : $2 \%$ ). Ces données concordent avec les pratiques françaises de 411 urologues et sexologues (Enquête PISTES 2002) dans lesquelles les bilans les plus prescrits sont biologiques (hormonal : $90 \%$, métaboliques: $74 \%$ ) devançant largement l'échodoppler (30\%), le test intracaverneux pharmacologiques $(22 \%)$, les explorations neurologiques $(11 \%)$, la rigidimétrie nocturne $(5 \%)$ et la cavernométrie $(1,5 \%)$.

\section{Vasculaire}

La quasi-disparition de la chirurgie vasculaire réparatrice (veineuse ou artérielle) du DE a réduit parallèlement les indications de l'imagerie médicale $[3,22,35,44]$. Ces examens sont très peu prescrits car ils ne modifient que très rarement la conduite à tenir thérapeutique.

Trois examens explorent la composante vasculaire périphérique $[18,22,35]$ : a) l'échodoppler pulsé pénien (apport artériel et drainage veineux), b) l'artériographie honteuse sélective (lit artériel pré et intrapénien), c) la cavernométrie-cavernographie (corps caverneux et verrouillage veino-caverneux). Leur fiabilité est très controversée $[18,26,35,37,49,52]$ pour des problèmes méthodologiques (manque de standardisation, de reproductibilité et de références) aggravés par leur aspect trop opérateurdépendant. Ces explorations ne doivent jamais être prescrites de première intention $[18,22,35,44]$. Elles sont sans valeur en l'absence de stimulation pharmacologique (la dose usuelle étant de $10 \mu \mathrm{g}$ de PGE1). La PGE1 intraurétrale à la dose $500 \mu \mathrm{g}$ (correspondant à $10 \mu \mathrm{g}$ en intracaverneux) peut être utilisée pour sensibiliser l'échodoppler car elle a un effet identique sur la vasodilatation artérielle et la vitesse maximale de débit systolique (RCP Vidal 2003 MUSE (8). Le sildénafil a également été proposé [50].

En pratique, l'échodoppler pulsé est l'examen vasculaire actuel "de référence" à la condition d'explorer aussi les gros vaisseaux périphériques dans le cadre du bilan d'une pathologie artérielle diffuse $[18,22,26,35,37,43,44,52]$. Les divers paramètres explorés (vitesse du pic systolique et du flux diastolique, temps d'accélération, diamètre artériel, index de résistance) orientent vers un défaut d'apport (athérosclérose) ou de maintien (fuite veinocaverneuse) mais ne font pas l'objet d'un consensus [18, 35, 48]. L'artériographie et/ou la cavernométrie ne sont indiquées que dans les exceptionnels cas de DE post-priapisme ou posttraumatique du sujet jeune pour lesquels une chirurgie est envisagée $[18,22,35,44]$.

\section{Rigidimétrie}

La mesure de la rigidité pénienne lors d'une stimulation pharmacologique par IIC (ou plus récemment oral) [35] ou d'une stimulation érotique visuelle a été abandonnée à la différence de l'enregistrement des érections physiologiques nocturnes qui cherche à distinguer les étiologies psychogènes ou organiques $[1,15,18,22,35,44]$. Ce test enregistre la durée et la rigidité des érections nocturnes en l'exprimant en pourcentage. Les pics de pression des érections nocturnes sont enregistrés par un rigidimètre informatisé idéalement couplé à un enregistrement parallèle du sommeil (les érections survenant lors de phases de sommeil paradoxal). L'exploration est considérée comme normale si le patient présente au moins une érection supérieure ou égale à $60 \%$ pendant au moins 10 minutes $[1,18$, 35].

Malheureusement, l'expérience clinique a montré plusieurs limites à cet examen : lourdeur méthodologique, coût de l'investissement et fiabilité limitée du fait de l'existence de faux négatifs (liés aux troubles du sommeil) et de faux positifs (certaines étiologies hormonales ou neurologiques) $[15,22,35,44]$. En conséquence, la présence d'érections nocturnes normales permet simplement d'affirmer l'intégrité des mécanismes vasculo-tissulaires locaux de l'érection. Les indications de la rigidimétrie sont très spécifiques et rares car elle n'est plus utilisée que dans quelques centres hyperspécialisés pour des problèmes médico-légaux, ou à visée de recherche (analyse de l'action pro au antiérectile d'agents pharmacologiques ou toxiques, évaluation de la capacité érectile post-prostatectomie) et dans les rares cas où il persiste un doute diagnostique avant une éventuelle chirurgie chez les sujets jeunes [18, 35, 44].

\section{Explorations neurophysiologiques}

De multiples explorations (mesure du temps de latence du réflexe bulbocaverneux, mesure de la vitesse de conduction du nerf dorsal de la verge, potentiels évoqués somes- 
thésiques ou cutanés sympathiques, EMG des corps caverneux) ont été proposées $[1,15,18,35,53,54]$. Leur but est de confirmer une participation neurogène et d'en préciser le niveau. Mais, comme pour les examens vasculaires, la validation de ces tests reste insuffisante. En pratique, ces examens ont peu d'indications en dehors de protocoles de recherche clinique ou de problèmes médicolégaux. Dans cette optique, la tomographie cérébrale par émission de positons apparaît comme une nouvelle voie d'exploration. L'étude récente chez le volontaire sain [53] a ainsi montré que l'installation ou non de l'excitation sexuelle dépendrait de l'activation ou la désactivation de centres cérébraux spécifiques (cognitif, motivationnel, émotionnel, physiologique) tandis que chez le sujet ayant une DE, l'apomorphine exercerait bien une action stimulatrice centrale [27].

\section{Divers}

La biopsie des corps caverneux, la mesure de la tension en oxygène, la pénographie isotopique ou l'imagerie nucléaire des corps caverneux restent toujours du domaine de la recherche sans application clinique actuellement [35].

\section{CONCLUSIONS}

La difficulté de reconnaître la cause précise de la DE jointe à l'efficacité remarquable des traitements pharmacologiques actuels a légitimement soulevé la question de l'intérêt de rechercher son étiologie. La réponse est clairement oui car le bilan à visée étiologique permet de reconnaître au niveau :

a) de l'individu : les étiologies bénéficiant d'une prise en charge spécifique, le profil de risque de DE pour optimiser la prise en charge diagnostique et thérapeutique, les patients à moins bon pronostic, les pathologies ou les situations à risque pour la santé méconnues ou insuffisamment prises en charge ;

b) de la population : le bilan d'un DE peut s'inscrire véritablement dans une démarche de santé publique dans la mesure où, d'une part, elle associe un dépistage de morbidités chroniques à des mesures de prévention et d'éducation d'une bonne santé, d'autre part, la prévention n'est pas une spécialité mais plus une manière de travailler visant à une promotion de la santé comme un état de bien-être sans se limiter à la seule absence de la maladie.

La double valeur du DE en tant que marqueur de la qualité de vie (DE maladie) et de symptôme clinique (DE symptôme) a profondément modifié son approche. L'existence ou non d'un retentissement sur la qualité de vie du patient (et du couple) conditionne la prescription d'un bilan complémentaire qui analysera la santé sexuelle et/ou physique et/ou psychique car le concept DE symptôme/maladie implique une prise en charge spécifique de ces états de santé. Elle explique : a) la nette réduction des investigations centrées sur l'organe effecteur pénien au profit d'une évaluation plus globale des multiples facteurs biomédicaux et environnementaux susceptibles d'affecter l'état de "bonne santé", b) le concept de profil de risque de DE qui implique que tout $D E$ est assimilable à une fenêtre s'ouvrant sur de nombreuses situations à risque (addictions, surmenage, surpoids...) et/ou des pathologies chroniques (cardiovasculaires, diabète, troubles de l'humeur, iatrogénie...). Ainsi, devant tout patient consultant pour un $D E$, il faut différencier le bilan :

a) de première intention : le seul bilan de pratique quotidienne vise à distinguer les cas simples ou plus compliqués qui nécessitent une prise en charge plus spécifique. Ce bilan de "débrouillage " s'inscrit dans une démarche éthique d'une médecine humaniste et de santé publique (agir sur les facteurs de risque modifiables, promotion de la "bonne santé"...) qui peut (et doit) être fait ou prescrit par tout soignant en premier contact quelle que soit sa qualification. II intègre une évaluation: 1) clinique cardiovasculaire et sexopsychologique systématiques minimales puis, selon les données cliniques, un avis complémentaire psychiatrique, urologique, neurologique, endocrinologique, etc.; 2) biologique minimal à la recherche d'anomalies cardiovasculaires, hormonales et urologiques.

b) de deuxième intention : à ne demander qu'en cas d'échec de la première approche et qui relève du soignant familiarisé à la prise en charge du DE (via surtout un test pharmacologique intracaverneux et/ou une sexothérapie) et/ou d'une autre spécialité d'organe.

c) de troisième intention : très rarement prescrit et relevant du domaine de I' "hyperspécialiste" du DE. Très schématiquement, l'âge permet de distinguer trois situations:

1) avant 35 ans, sauf cas particulier (antécédent familial ou personnel cardiovasculaires, facteurs de risque cardiovasculaire, DE post-traumatique), le DE reflète d'abord, un trouble psychologique ou relationnel nécessitant une évaluation sexo-psychologique (voire psychiatrique) d'autant plus qu'il est primaire et/ou associé à d'autres troubles sexuels (un examen de médecine générale suffit pour le versant "somatique");

2) après 35 ans, un bilan somatique s'impose parallèlement car le DE est, d'abord, un signe vasculaire (bilan clinique et biologique) puis de diverses pathologies chroniques connues ou non, souvent présentes à l'origine du concept de profil de risque ;

3) après $\mathbf{5 0}$ ans, il faut rajouter un bilan urologique et hormonal ("andropause" à évoquer de principe). Dans tous les cas, il faut distinguer le sujet en bonne santé apparente, sans antécédent ni facteur de risque particulier (personnels ou familiaux cardiovasculaires, diabétiques...) régulièrement suivi par son médecin généraliste du sujet non ou mal suivi chez lequel le bilan (et la prise en charge) d'éventuels facteurs de risque cardiovasculaires est indispensable. 


\section{REFERENCES}

1. ARVIS G., AMARENCO G., AMER M., et al. : Les explorations paracliniques de dysfonctions sexuelles. In: Arvis $G$. ed. Andrologie. Paris, Maloine, 1991, (tome III), 130: 1815-1906.

2. ARRUDA-OLSON A., MAHONEY D.W., NEHRA A., LECKEL M., PELLIKAA P.A. : Cardiovascular effects of sildenafil during exercise in men with known or probable coronary heart disease. A randomized crossover trial. J. Am. Med. Ass., 2002, 287 : 719-725.

3. BONDIL P., BLACHERE P. : Le traitement de l'insuffisance érectile : quels sont les objectifs et les méthodes ? Prog. Urol., 1998, $8: 17-31$

4. BONDIL P. : Quelles investigations faut-il demander ? Impact Médecin Hebdo, 1999, 471 : IX-X.

5. BONDIL P., DELAVIERRE D. : Facteurs étiologiques et facteurs de risque. In : Bondil P. ed. La dysfonction érectile. Paris, John Libbey Eurotext, 2003 : 39-62.

6. BONDIL P: Gestion de l'étape clinique. In : Bondil P. ed. La dysfonction érectile. La dysfonction érectile. Paris, John Libbey Eurotext, 2003 : 99-118.

7. BRENOT P. : La sexologie. Paris, PUF, 1994.

8. BUVAT J., BUVAT-HERBAUT M. : La prise en charge d'inspiration sexologique. In: Arvis G. ed. Andrologie. Paris, Maloine, 1991, (tome III), $139: 20392040$.

9. BUVAT J., LEMAIRE A. : Endocrine screening in 1022 men with erectile dysfunction : clinical significance and cost-strategy. J. Urol., 1997, $158: 1764-1767$

10. BUVAT J., RATAJCZYK J., LEMAIRE A. : Les problèmes d'érection : une souffrance encore trop souvent cachée. Andrologie, 2002, $12: 73-83$.

11. CHEVRET-MEASSON M. : Les différentes prises en charge psychothérapiques et sexothérapiques. In : Bondil P. ed. La dysfonction érectile. Paris, John Libbey Eurotext, 2003 : 179184.

12. CHUN J., CARSON C.C. : Physician-patient dialogue and clinical evaluation of erectile dysfunction. Urol. Clin. North Am., 2001, $28: 249-258$.

13. Comité Consultatif National d'Ethique (CCNE), Rapport $n^{\circ} 62$ : Médicalisation de la sexualité : le cas sildénafil; réponse au secrétaire d'état à la santé, novembre 1999 (rapporteurs: Beaulieu E.E., Montagut J.) www.ccne-ethique.org.

14. CUSSENOT O., TEILLAC P. : Le cancer de prostate. Paris, John Libbey Eurotetx, 1999.

15. CUZIN B. : Intérêt des examens complémentaires dans le bilan d'une insuffisance érectile. In : Giami A., Pietri L. eds. Les traitements de l'impuissance. Paris, La Documentation française, $1999: 105-112$.

16. CUZIN B., GIULIANO F., JAMIN C., et al. : Diagnostic, traitement et surveillance de l'hypogonadisme de survenue tardive chez l'homme : Recommandations officielles de l'International Society for the study of the aging male (ISSAM) et commentaires. Andrologie, 2003, $13: 331-348$.

17. DEBUSK R., DRORY Y., GOLDSTEIN I., et al : Management of sexual dysfunction in patients with cardiovascular disease : recommendations of the Princeton Consensus Panel. Am. J. Cardiol., 2002, $86: 175-181$.
18. DELAVIERRE D., CUZIN B., GIULIANO F. : Quelle place reste-t-il aux examens complémentaires dans l'exploration de l'insuffisance érectile? Sexologies, 1999, $9: 23-26$.

19. DELAVIERRE D. : Epidémiologie de la dysfonction érectile

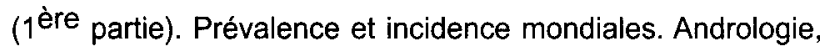
2002, $12: 167-185$.

20. DERBY C.A., MOHR B.A., GOLDSTEIN I., FELDMAN H.A., JOHANNES C.B., McKINLAY J.B. : Modifiable risk factors and erectile dysfunction : can lifestyle changes modify risk ? Urology, 2000, $56: 302-306$.

21. ELHANBLY S., SCHOOR R., ELMOGY M. et al. : What nonresponse to intracavernous injection really indicates : a determination by quantitative analysis. J. Urol., 2002, 167 : 192196.

22. European Association of Urology (EAU) : Guidelines on erectile dysfunction. 2003.

23. FELDMAN H.A., GOLDSTEIN I., HATZICHRISTOU D.G., KRANE R.J., McKINLAY J.B. : Impotence and its medical and psychosocial correlates : results of the Massachusetts Male Aging Study. J. Urol., 1994, $151:$ 54-61.

24. GIAMI A.: Sexual health : The emergence, development and diversity of a concept. Ann. Rev. Sex Res., 2002, 13 : 1-35.

25. GIULIANO F., CHEVRET-MEASSON M., TSATARIS A. et al : Prévalence de l'insuffisance érectile en France : résultats d'une enquête épidémiologique menée auprès d'un échantillon représentatif de 1004 hommes. Prog. Urol., 2002, 12 : 260-267.

26. GOLUBINSKI A.J., SIKORSKI A. : Usefulness of power Doppler ultrasonography in evaluating erectile dysfunction. $\mathrm{Br}$. J. Urol. Int., 2002, 89 : 779-782.

27. HAGEMAN J.H., BERDING G., BERGH S. et al. : Effects of visual sexual stimuli and apomorphine SL on cerebral activity in men with erectile dysfunction. Eur. Urol., 2003, 43 : 412420.

28. HAIAT R., LEROY G. : Facteurs de risque cardiovasculaire: les enseignements des grands essais cliniques. Coll. Médecine et preuves. Paris, Frison-Roche, 2003.

29. HURET M., OLIVIER V. : Peut-on évaluer les psychothérapies ? L'Express, 2004, 2747 : 100-101.

30. KIRBY M. : Erectile dysfunction and vascular disease. Oxford, Blackwell Publishing Ltd, 2003.

31. KLONER R.A., MULLIN S.H., SHOOK T. et al. : Erectile dysfunction in the cardiac patient : how common and should we treat? J. Urol., 2003, 170 : S43-\$40.

32. LEJEUNE H. : Le déficit androgénique lié à l'âge chez l'homme : terminologie, épidémiologie. Andrologie, 2002, 12 : 1116.

33. LEWIS R., HATZICRISTOU D., LAUMANN E.O., MCKINLAY $\mathrm{J}$. : Epidemiology and natural history of erectile dysfunction : risk factors including iatrogenic and aging. In : Jardin A., Wagner G., Khoury S., Padma-Nathan H., Rosen R. eds. Erectile dysfunction. First International Consultation on Erectile Dysfunction, Paris, juillet 1999. Oxford, Health Publication Ltd, 2000.

34. MEULEMAN E., BRODERICK G., MENG TAN H., MONTORSI F., SHARLIP I., VARDI Y.: Clinical evaluation and the doctor-patient dialogue. In : Jardin A., Wagner G., Khoury S., Giuliano F., Padma-Nathan H., Rosen R. eds. Erectile Dysfunction (2000) First International Consultation on Erectile Dys- 
function. Oxford, Health Publication Ltd, 2000 : 117-138.

35. MEULEMAN E.J.H.: Investigations in erectile dysfunction. Curr. Opin. Urol., 2003, $13: 411-416$.

36. MEULEMAN E.J., MULDERS P.F. : Erectile function after radical prostatectomy: a review. Eur. Urol. 2003, 43 : 95-101

37. MILLS R.D., SETHIA K.K. : Reproductibility of penile arterial colour duplex ultrasonography. Br. J. Urol., 1996, 78, 109-112.

38. MONCADA I. : LUTS: a risk factor for sexual dysfunction in the BPH patient. Eur. Urol., 2003 (suppl. 2) : 3-8.

39. MONTORSI F., BRIGANTI A., SALONIA A. et al. : Erectile dysfunction prevalence, time of onset and association with risk factors in 300 consecutive patients with acute chest pain and angiographically documented coronary artery disease Eur. Urol., 2003, $44:$ 360-365.

40. MONTORSI F., BURNETT A.L. : Erectile dysfunction after radical prostatectomy. Br. J. Urol. Int., 2004, 93 : 12.

41. NINOMIYA J.K., CRIQUI G., KATZ L.D., et al. : Association of the metabolic syndrom with history of myocardial infarction and stroke in the Third National Health and Nutrition Examination Survey. Circulation, 2003, 109 : 42-46.

42. PASINI W. : Est-ce que traiter l'érection signifie guérir l'impuissance ? Andrologie, 2003, $13: 207-208$

43. O'LEARY M.P., BAUM N.H., PRICE D.K., et al. : 2001 american urological association gallup survey : changes in physician practice patterns, satisfaction with urology, and treatment of prostate cancer and erectile dysfunction. J. Urol., 2002, 168 : 649-652.

44. Recommandations de la Première Consultation Internationale sur la Dysfonction Erectile (ICED), Paris 1999. Jardin A., Wagner G., Khoury S., Giuliano F., Goldstein I., PadmaNathan H., Rosen R. eds. Oxford, Health Publications Ltd, 2000.

45. RICHARD F., LUKACS B., JARDIN A., et al. : Résultats d'une enquête épidémiologique menée chez les des hommes âgés de 50 à 80 ans étudiant troubles mictionnels, qualité de vie et fonction sexuelle. Progr. Urol., 2001, 11 : 250-263.

46. ROSEN R.C. : Psychogenic erectile dysfunction. Classification and management. Urol. Clin. North Am., 2001, 28 : 269278.

47. ROUMEGUIERE T., WESPES E., CARPENTIER Y., HOFFMAN P., SCHULMAN C.C. : Erectile dysfunction is associated with a high prevalence of hyperlipidemia and coronary heart disease risk. Eur. Urol., 2003, 44 : 355-359.

48. SADOVSKY R., MILLER T., MOSKOWITZ M., HACKETT G. : Efficacy and tolerance of citrate of sildenafil (Viagra $(B)$ ): three years after. Int. J. Clin. Pract., 2001, 55 : 115-130.

49. SLOB A.K., CORNELISSEN S., DOHLE G.R., GIJS L., VAN DER WERFF TEN BOSCH J.J. : The limited practical value of color Doppler sonography in the differential diagnosis of men with erectile dysfunction. Int. J. Impot. Res., 2002, 14 : 201203.

50. SPEEL T.G., BLEUMER I., DIEMONT W.L., et al. : The value of sildenafil citrate as mode of stimulation in pharmaco-penile duplex ultrasonography. Int. J. Impot. Res., 2001, 13 : 189191.

51. SPEEL T.G.W., Van LANGEN H., MEULEMAN E.J.H. : The risk of coronary heart disease in men with erectile dysfunction. Eur. Urol., 2003, 44 : 366-371.

52. SPEEL T.G., Van LAZNGEN H., WIJKSTRA H., MEULEMAN E.J. : Penile duplex ultrasonography revisited : revalidation of the parameters of the cavernous arterial response. J. Urol., 2003, $169: 216-220$.

53. STOLERU S. : Approche du désir et de l'excitation sexuels par l'imagerie cérébrale fonctionnelle. Sexologies, 2002, 11 : 63-68.

54. VARDI Y., GRUNENWALD I., SPRECHER F. : The role of corpus cavernosum electromyography. Curr. Op. Urol., 2000, 10 : 635-638.

55. VIRAG R., BECHER E., CARRIER S., et al. : Local pharmacological treatment modalities. In : Jardin A., Wagner G., Khoury S., Padma-Nathan H., Rosen R. eds. Erectile dysfunction. First International Consultation on Erectile Dysfunction, Paris, juillet 1999. Oxford, Health Publication Ltd, 2000.

56. WAGNER G., CLAES H., COSTA P., et al. Lygon Arms Group. A shared care approach to the management of erectile dysfunction in the community. Int. J. Impot. Res., 2002, 14 : 189194.

Manuscrit reçu : avril 2004 ; accepté juin 2004.

\section{ABSTRACT}

What investigations should be ordered by non-specialists in a patient consulting for erectile dysfunction?

\section{Pierre BONDIL}

The real difficulty of a precise diagnosis and the efficacy of currently available drugs have not eliminated the need for an aetiological work-up of erectile dysfunction (ED). This work-up is essential in order to identify: a) aetiologies requiring specific management, b) the ED risk profile, c) patients with a less favourable prognosis, d) situations associated with a health risk and/or untreated or poorly managed diseases. The approach to ED has been profoundly modified by the demonstration that ED is a relevant marker of quality of life (ED disease) and is also a clinical symptom (ED symptom) of multiple high-risk situations or chronic diseases. The presence or absence of a repercussion on the patient's (and the couple's) quality of life therefore guides the assessment and management of the patient's sexual and/or physical and/or mental health.

This concept of ED disease/ED symptom explains the decreased use of exclusively penis-based investigations in favour of global evaluation of the multiple biomedical and environmental factors able to affect the state of "good health". In practice, the development of ED is never purely benign, as it requires a preliminary assessment (always clinical and often laboratory) accessible to any first-line physician regardiess of his or her qualification. The objective of this initial assessment is to distinguish 
simple cases (that can be treated by the great majority of doctors) from more complex cases.

In the presence of specific abnormalities and/or failure of first-line management, a more specialized assessment, possibly by a specialist in the management of ED, may be indicated. Very schematically: 1) before the age of 35 years, ED is primarily due to a psychological/relational disorder, which requires sexological-psychological (or even psychiatric) assessment, especially when ED is primary or longstanding, 2) after the age of 35 years, ED is also and primarily a sign of vascular disease and then various chronic morbidities hence the need for an associated somatic assessment, 3 ) after the age of 50 years, a urological and hormonal assessment ("andropause") must be added. Systematic analysis of the risk profile therefore allows the assessment of any man consulting for ED to be integrated into an ethical approach providing a service for the patient, as well as an active and collective public health approach.

Key-Words: erectile dysfunction, diagnosis, assessment, investigation, aetiology, cardiovascular screening 